\title{
Low yield for non-targeted biopsies of the stomach and esophagus during elective esophagogastroduodenoscopy
}

\section{(c) (1) $\Theta$}

Authors

Michael K. Dougherty ${ }^{1}$, Phillip P. Santoiemma², Andrew T. Weber ${ }^{3}$, David C. Metz ${ }^{4}$, Yu-Xiao Yang ${ }^{5}$

Institutions

1 Division of Gastroenterology, Department of Medicine, University of North Carolina, Chapel Hill, North Carolina, United States

2 Department of Medicine, Northwestern University Feinberg School of Medicine, Chicago, Illinois, United States

3 Department of Medicine, University of California Los Angeles, Los Angeles, California, United States

4 Division of Gastroenterology, Department of Medicine, Hospital of the University of Pennsylvania, Philadelphia, Pennsylvania, United States

5 Division of Gastroenterology, Department of Medicine, Perelman School of Medicine at the University of Pennsylvania, Philadelphia, Pennsylvania, United States

submitted 21.11.2016

accepted after revision 31.7.2017

\section{Bibliography}

DOI https://doi.org/10.1055/s-0043-119791 |

Endoscopy International Open 2017; 05: E1268-E1277

(c) Georg Thieme Verlag KG Stuttgart · New York

ISSN 2364-3722

Corresponding author

Yu-Xiao Yang, MD, MSCE, FACP, Division of

Gastroenterology, Department of Medicine, Perelman

School of Medicine at the University of Pennsylvania, 423

Guardian Drive, 733 Blockley Hall, Philadelphia, PA 19104

yangy@pennmedicine.upenn.edu

\section{ABSTRACT}

Background and study aims Biopsies of non-specific mucosal findings are often performed during esophagogastroduodenoscopy (EGD). We sought to determine the prevalence and clinical utility of non-targeted biopsies of the stomach and esophagus.

Patients and methods We conducted a retrospective review of 949 outpatient EGDs performed at a US tertiary referral center. Non-targeted biopsies of the stomach were defined as either "normal" or "mild" to "moderate" "erythema" or "inflammation" without other endoscopic features. Non-targeted biopsies of the esophagus and gastroesophageal junction (GEJ) were defined as endoscopically "normal" mucosa. The primary outcome was the proportion of non-targeted biopsies resulting in "definite management change." Secondary outcomes included histopathologic diagnoses of Helicobacter pylori, intestinal metaplasia and esophageal eosinophilia.

Results Of 949 EGDs, 332 (35.0\%, 95\% Cl 31.9-38.1\%) had a non-targeted biopsy taken at any site. Erythema in the gastric body and antrum was biopsied at a rate of 83 $86 \%$, while biopsies of "normal"-appearing mucosa occurred at rates from $3 \%$ (GEJ) to $15 \%$ (body and antrum). The percentage of non-targeted biopsies that led to definite management change ranged from $5 \%$ in the GEJ and esophagus to $9 \%$ in the antrum, but did not significantly differ by mucosal appearance. Multivariable regression analyses suggested associations of language and age $>50$ with management change from non-targeted gastric biopsy.

Conclusions Non-targeted biopsies of the stomach and esophagus led to definite management change in a small proportion of patients. Further studies are needed to identify patient and/or endoscopic characteristics and techniques to improve the yield of this practice.

\section{Introduction}

Biopsies of non-specific mucosal findings or even normal mucosa in the stomach and esophagus are often performed during esophagogastroduodenoscopy (EGD). While the risk of obtaining cold biopsies of the upper gastrointestinal tract is negligible, such a practice entails substantially increased procedure cost and associated anatomic pathology cost. Payers including the United States General Accountability Office have recently expressed concerns regarding the potential overuse of EGD biopsies [1]. However, data regarding the precise prevalence and clinical utility of biopsies of normal-appearing mucosa are scarce [2]. 
Gastric biopsy is most often performed during the evaluation of dyspepsia, to diagnose Helicobacter. pylori (HP) or perhaps gastric intestinal metaplasia (GIM), and rarely occult dysplasia or neoplasia. However, histopathology from non-targeted biopsies correlates poorly with symptoms [3-5]. Prospective random biopsy protocols have not consistently demonstrated a symptomatic benefit for HP eradication in functional dyspepsia, the clinical effect size of such eradication is small [5], and the cost-effectiveness of routine biopsies of normal-appearing mucosa among patients with dyspepsia remains uncertain [6]. Further, even rigorous gastric biopsy protocols such as the Updated Sydney Protocol may not be sufficiently sensitive for premalignant lesions $[7,8]$, and rarely yield dysplasia or neoplasia [9-11]. As such, there is generally no consensus on screening for and surveillance of premalignant lesions in populations with low prevalence of gastric cancer [12-15].

Practice guidelines are vague about gastric biopsy practice in the undifferentiated patient presenting for EGD. Some indicate that "biopsy specimens should be obtained for $\mathrm{H}$. pylori" without much indication of region or number [17], or even omit recommendations on biopsy practice altogether 18]. The most recent American Gastroenterological Association (AGA) guidelines endorse the Sydney Protocol for the purposes of establishing $H$. pylori status in "normal-appearing" gastric mucosa, which includes mucosa noted to have "erythema." $[2,19]$ Recent European guidelines have recommended a strategy for detection of GIM modeled after the Sydney protocol that explicitly includes non-targeted biopsies [20], but it is unclear to which subjects this should be initially applied.

In the esophagus, excluding the controversial practice of biopsying the "irregular Z-line," non-targeted biopsies are taken most often to detect eosinophilic esophagitis (EoE). American guidelines for endoscopy in GERD recommend either against non-targeted biopsies [21], or only in those patients with "troublesome dysphagia" [22], to look for EoE. However, EoE guidelines leave significant room for interpretation regarding whom to biopsy and with what protocol [23]. Reliable estimates of EoE incidence in completely normal-appearing adult esophagi are still elusive [24], but it is likely rare in adults without dysphagia [25].

Given the lack of data regarding the clinical yield of non-targeted biopsy during EGD and the resultant variation in practice, this is an important target for optimizing value in healthcare. Because non-targeted biopsy of the duodenum was recently examined [26], the current study seeks to characterize this practice in the stomach and esophagus.

\section{Patients and methods}

\section{Design}

We retrospectively reviewed a random sample of outpatient EGDs performed within the University of Pennsylvania Health System (UPHS), United States for a descriptive study of the practice of non-targeted biopsy during EGD. This study was approved by the University of Pennsylvania Institutional Review Board.

\section{Data Source}

We reviewed the UPHS Olympus endoscopy database to identify patients undergoing EGD at three UPHS-affiliated gastroenterology practices during the study period. Three authors then extracted all remaining patient characteristics from EPIC, the electronic medical record system used by over 60 UPHS ambulatory sites.

\section{Study patients}

We extracted all elective outpatient EGDs performed at the 3 UPHS gastrointestinal endoscopy sites between 7/1/2013 to $12 / 31 / 2013$. Based on an analysis of the first 150 patients, we calculated that a sample size of 1000 patients would yield an acceptable confidence interval width of $13 \%$ (around a point estimate of $16 \%$ ) for the primary outcome of management change, in the gastric body. In order to avoid bias, we used the RAND function in Microsoft Excel to ensure that our study patients represented a random sample of all patients undergoing EGD during the study period. Exclusion criteria included subjects aged $<18$ years, aborted procedures, or any subsequent EGD's on the same subject within the study period.

\section{Variables}

The unit of analysis was the EGD procedure, and only the first EGD for each patient in the study period was included. Indications for EGD were recorded only from the endoscopy report, although clinic notes were reviewed for other patient characteristics as well as to determine management changes. Major comorbidities recorded were active malignancy in the last year, AIDS (CD4 count $<200$ in last year), history of organ transplant, otherwise significantly immunosuppressed, history of gastric or esophageal surgery, cirrhosis or portal hypertension, bleeding diathesis or non-suspended therapeutic anticoagulation (including dual antiplatelet therapy), and inflammatory bowel disease. Indications were taken from the endoscopy report as one of the following: dyspepsia, chest pain, GERD, anemia, weight loss, recent gastrointestinal bleeding, nausea/vomiting, change in bowel habits, dysphagia/odynophagia, bloating/early satiety, Barrett's surveillance, surveillance of other condition (e.g. EoE, familial adenomatous polyposis, etc.), follow-up of previous endoscopy (e.g. ulcer healing, previous endoscopic mucosal resection [EMR]), follow-up of noninvasive imaging finding, variceal screening, bariatric or other pre-operative indication, planned intervention (e.g. dilatation, EMR, $\mathrm{pH}$ probe placement, etc.), part of advanced endoscopy procedure, or "other."

A "non-targeted" biopsy of the stomach was defined as from mucosa described in the EGD report as either "normal" or "mild" to "moderate" "erythema," "inflammation," or "gastritis" without other endoscopic features (such as nodularity). This definition was intentionally created to examine the extent to which mucosal appearance is related to outcome, if such appearance is non-specific. It was also chosen to reflect the recent AGA Institute Technical Review on biopsies to evaluate dyspepsia, which classified mucosal "erythema" among "normal" biopsies [2]. Non-targeted biopsies of the tubular esophagus 
- Table 1 Types of management changes by region.

\begin{tabular}{|c|c|}
\hline Management change ${ }^{1}$ & n (\%) \\
\hline \multicolumn{2}{|l|}{ Stomach } \\
\hline \multicolumn{2}{|l|}{ Definite management change $(n=28)$} \\
\hline Treatment for $\mathrm{HP}^{2}$ & $23(82 \%)$ \\
\hline Treatment for Helicobacter heilmannii & $1(4 \%)$ \\
\hline Investigation of HP status (for GIM) & $2(7 \%)$ \\
\hline Surveillance EGD for gastric mapping (for GIM) & $2(7 \%)$ \\
\hline Follow-up with additional provider (oncologist) & $1(4 \%)$ \\
\hline \multicolumn{2}{|c|}{ Management change of "uncertain significance" $(n=24)$} \\
\hline Start, increase or continue PPI or H2RA & $17(71 \%)$ \\
\hline $\begin{array}{l}\text { Additional laboratory workup or referral based on } \\
\text { "negative" pathologic finding }\end{array}$ & $3(13 \%)$ \\
\hline $\begin{array}{l}\text { Additional laboratory workup based on "positive" } \\
\text { pathologic finding (eosinophilia) }\end{array}$ & $1(4 \%)$ \\
\hline “Possible” repeat endoscopy in 2-3 years (for GIM) & $2(8 \%)$ \\
\hline Stop NSAID & $2(8 \%)$ \\
\hline Additional visit with endoscopist (to discuss GIM) & $1(4 \%)$ \\
\hline \multicolumn{2}{|l|}{ Gastroesophageal junction } \\
\hline \multicolumn{2}{|l|}{ Definite management change $(n=1)$} \\
\hline Referral for repeat intervention (for dysplasia) & $1(100 \%)$ \\
\hline \multicolumn{2}{|l|}{ Esophagus } \\
\hline \multicolumn{2}{|l|}{ Definite management change $(n=4)$} \\
\hline Commencement of topical steroid & $2(50 \%)$ \\
\hline Discontinuation of steroid, commencement of PPI $\dagger$ & $1(25 \%)$ \\
\hline Re-restriction of diet & $1(25 \%)$ \\
\hline \multicolumn{2}{|c|}{ Management change of "uncertain significance" $(n=4)$} \\
\hline Continue current therapy & $1(25 \%)$ \\
\hline "Optional" increase in dietary restrictions & $1(25 \%)$ \\
\hline Introduction of new foods & $1(25 \%)$ \\
\hline Start PPI (for some microscopic inflammation) & $1(25 \%)$ \\
\hline \multicolumn{2}{|c|}{$\begin{array}{l}\text { HP, H. Pylori; GIM, gastric intestinal metaplasia; PPI, proton pump inhibitor; } \\
\text { H2RA, histamine } 2 \text { receptor antagonist; NSAID, non-steroidal anti-inflam- } \\
\text { matory drug } \\
{ }^{1} \text { More than one possible per patient and biopsy. } \\
{ }^{2} \text { Where biopsy revealed distal-predominant eosinophilia in a patient who } \\
\text { had never had a PPI trial to determine possible PPI-responsive eosinophilia. }\end{array}$} \\
\hline
\end{tabular}

and GEJ were those performed on endoscopically "normal" mucosa (without any erythema or esophagitis, and without an irregular Z-line). Other lesions elsewhere in the stomach (such as polyps, "severe" inflammation, erosions or ulcers) were noted but did not affect non-targeted classification, as long as non-targeted biopsies were not taken from these lesions. The primary outcome was the proportion of non-targeted biopsies where the pathology (not the endoscopic findings) directly resulted in a "definite management change." This was defined as a recommendation to start or discontinue a prescription medication, undergo an additional procedure or test, or see an additional specialist in response to a new, definitive histologic finding from the non-targeted biopsy ( $\triangleright$ Table 1 ). We also identified biopsies that resulted in "definitely no management change" if explicitly indicated in the medical records. A small proportion of biopsies could not be classified in either of the above categories. For these, "unclear management changes" captured instances in which the medical records contained no information to allow the determination of management change. In addition, management changes "of uncertain significance" included titration of acid suppression or other interventions that were not clearly related to the histopathology results ( $>$ Table 1).

\section{Statistical analysis}

We calculated the frequency with which non-targeted biopsy was performed, the proportions of the non-targeted biopsies with $95 \%$ confidence interval $(\mathrm{Cl})$ that were associated with the respective management change categories, as well as the prevalence of clinically important histological changes such as HP infection, intestinal metaplasia (IM), and esophageal eosinophilia. We also conducted a secondary, exploratory analysis of patient, procedure, and mucosal characteristics associated with the outcomes of management change and the specific histopathologic findings of HP and GIM. The examined variables included age, sex, preferred language, race, HP status, history of prior EGD, the indications for EGD, the recorded comorbidities, and the presence of mild erythema, duodenitis, or peptic ulcer on endoscopy. Variables were screened in bivariable logistic regression using a cutoff of $P<0.2$. Surviving variables that were conceptually distinct were modeled with multivariable logistic regression with no more than one covariate per five outcomes, and then further reduced using likelihood ratio tests with a cutoff of $P<0.05$. The identified significant variables were then modeled using log-binomial regression to obtain adjusted prevalence ratios. The low number of outcomes prohibited analyses of esophagus, GEJ and gastric subregions, as well as inclusion of more than 2 independent variables per outcome variable. We conducted a sensitivity analysis of the regression models with the outcome of "any management change," both to control for any possible subjectivity in the determination of "definite" management change as well as allow for additional independent variable detection by increasing the number of outcomes. We also conducted a sensitivity analysis separating those subjects whose non-targeted gastric biopsies had "erythema" versus those with "normal mucosa only." All statistical analyses were performed with STATA 13 (STATA Corp., College Station, Texas, United States).

\section{Results}

The patient sample characteristics, including demographics, are displayed in $>$ Table 2 . 
- Table2 Demographics and clinical characteristics.

Age, years, mean (standard deviation) ${ }^{1}$

Male, $\mathrm{n}(\%)$ $437(46 \%)$

Last EGD, n (\%)

- In past 5 years $525(55 \%)$

- Never $233(25 \%)$

Comorbidities, n (\%)

- Active malignancy $88(9.3 \%)$

- History of organ transplant $25(2.6 \%)$

- Otherwise severely immunosuppressed $28(3.0 \%)$

- History of gastric or esophageal surgery $91(9.6 \%)$

- Cirrhosis or portal hypertension $75(7.9 \%)$

- Bleeding diathesis or anticoagulated $26(2.7 \%)$

- IBD or other severe inflammatory luminal disorder $49(5.2 \%)$

- No relevant comorbidity $612(64 \%)$

H. pylori status in past year ${ }^{2}$

$$
\text { - Positive }
$$

- Negative $37(3.9 \%)$

Indications ${ }^{3}$

- Dyspepsia/Abdominal pain 233

- Nausea/vomiting 60

- Bloating/early satiety

- Anemia/concern for gastrointestinal bleed

- Weight loss

- Change in bowel habit

- Dysphagia/odynophagia

- GERD/chest pain

- Barrett's surveillance

- Surveillance of other condition ${ }^{4}$

- Follow-up of previous endoscopy

- Follow-up of imaging finding

- Planned procedure or advanced endoscopy

- Variceal screening

- Preoperative (e.g. bariatric)

60

26

80

19

69

161

223

105

89

117

36

73

60

EGD, esophagogastroduodenoscopy; GERD, gastroesophageal reflux disease;

${ }^{1}$ Range $18-100$ years old

${ }^{2}$ Includes serology, stool antigen, breath test or biopsy-based test.

${ }^{3}$ May overlap

${ }^{4}$ Non-Barrett's, most often esophageal eosinophilia or familial adenomatous polyposis.

${ }^{5}$ Most often history of Barrett's ablation, occasionally for ulcer-healing, etc.

\section{Biopsy practice}

Nine hundred forty-nine randomly selected EGDs performed by 44 difference endoscopics were included in the analysis. There was substantial variation in the practice of obtaining non-targeted biopsies between endoscopists. Specifically, the median proportion of normal mucosa biopsied by individual endoscopist was $0 \%$ for the esophagus (inter-quartile range: $0-$ $14 \%$ ), $12 \%$ for the gastric body (inter-quartile range: $0-28 \%$ ), and $24 \%$ for the antrum (inter-quartile range: $10-50 \%$ ).

Of the 949 EGDs, 332 (35.0\%, 95\% Cl 31.9-38.1\%) had a non-targeted biopsy taken at any site. The frequency of nontargeted biopsies varied by endoscopic features and anatomic location ( $\triangleright$ Table 3 ). Erythema in the gastric body and antrum were most likely to be biopsied (83-86\%), while a normal GEJ and fundus were least likely (3\%). Frequency of biopsy varied by indication per region as expected, with esophageal-related indications resulting in more biopsies in the tubular esophagus or GEJ, and dyspepsia-related symptoms resulting in more biopsies of the stomach ( $\triangleright$ Supplemental Table 1 ). Of the normal-appearing GEJs biopsied, 13/19 were for surveillance of Barrett's (for a history of dysplasia, now status-post radiofrequency ablation), with the remainder during EGD's for reflux (3), abdominal pain/dyspepsia (2), dysphagia (1), and followup of eosinophilia (1). Dyspepsia-related indications led to more frequent non-targeted biopsies of the stomach largely from an increased tendency to biopsy "normal" stomach, as stomach "erythema" was biopsied at a high rate regardless of indication.

\section{Effect on management}

Regarding the primary outcome, the vast majority of non-targeted biopsies resulted in no management change ( $\triangleright$ Table 4 ). Biopsies of "normal" esophagus and GEJ least frequently impacted management (5\%). Biopsies of erythema in the fundus most frequently impacted management, though the small total number of fundus biopsies made this estimate less reliable $(\mathrm{Cl}$ 7-70\%). Furthermore, no definite management change based on a non-targeted biopsy of the fundus occurred without being based also on a non-targeted biopsy from the body or antrum (e. g. HP detected in both specimens). Management change occurred in $9 \%$ of antral non-targeted biopsies regardless of the presence of "erythema" or "normal" mucosa, and in $6 \%$ of "normal" mucosal biopsies versus $10 \%$ of "erythema" biopsies in the gastric body. These differences were not statistically significant by chi-square test $(P=0.35$ and $P=0.97$, respectively). Across all regions, the secondary outcome of "no management change" ranged from $67 \%$ to $83 \%$. Changes of "uncertain significance" were recorded in $5 \%, 0 \%, 9 \%, 4 \%$, and $9 \%$ of non-targeted biopsies from the esophagus, GEJ, fundus, body, and antrum-pylorus, respectively, with the remainder "unclear" based on the medical record.

Management changes in the stomach resulted largely from $\mathrm{HP}$ and/or IM, in the esophagus from eosinophilia, and in the GEJ from IM (i.e. Barrett's esophagus, see $>$ Table 1). Twentythree of 28 (82\%) definite management changes from biopsies of the stomach demonstrated HP, none of which were in the 
- Table 3 Frequency of non-targeted biopsy by endoscopic features and anatomic location.

\begin{tabular}{|c|c|c|c|c|c|c|c|c|}
\hline \multirow[b]{2}{*}{ Endoscopic features } & \multirow{2}{*}{$\begin{array}{l}\text { Esophagus } \\
\text { Normal } \\
(n=644)\end{array}$} & \multirow{2}{*}{$\begin{array}{l}\text { GEJ } \\
\text { Normal } \\
(n=673)\end{array}$} & \multicolumn{2}{|l|}{ Fundus } & \multicolumn{2}{|l|}{ Body } & \multicolumn{2}{|c|}{ Antrum-pylorus } \\
\hline & & & $\begin{array}{l}\text { Normal } \\
(n=825)\end{array}$ & $\begin{array}{l}\text { Erythema } \\
(n=25)^{*}\end{array}$ & $\begin{array}{l}\text { Normal } \\
(n=760)\end{array}$ & $\begin{array}{l}\text { Erythema } \\
(n=84)^{*}\end{array}$ & $\begin{array}{l}\text { Normal } \\
(n=639)\end{array}$ & $\begin{array}{l}\text { Erythema } \\
(n=172)^{1}\end{array}$ \\
\hline $\begin{array}{l}\text { Number biopsied, n } \\
(\%, 95 \% \mathrm{Cl})\end{array}$ & $\begin{array}{l}73 \\
(11,9-14)\end{array}$ & $\begin{array}{l}19 \\
(3,2-4)\end{array}$ & $\begin{array}{l}24 \\
(3,2-4)\end{array}$ & $\begin{array}{l}9 \\
(36,18-57)\end{array}$ & $\begin{array}{l}117 \\
(15,13-18)\end{array}$ & $\begin{array}{l}72 \\
(86,76-92)\end{array}$ & $\begin{array}{l}97 \\
(15,12-18)\end{array}$ & $\begin{array}{l}142 \\
(83,76-88)\end{array}$ \\
\hline
\end{tabular}

$\mathrm{Cl}$, confidence interval; GEJ, gastroesophageal junction

${ }^{1}$ The number of subjects with erythema of any gastric region was $n=201$, and 170 ( $85 \%$ ) had a non-targeted gastric biopsy. 118 ( $19 \%$ ) of 626 subjects with no gastric abnormality were biopsied.

- Table 4 Clinical and histopathologic outcomes of non-targeted biopsies during EGD.

\begin{tabular}{|c|c|c|c|c|c|c|c|c|c|c|c|}
\hline \multirow[b]{2}{*}{$\begin{array}{l}\text { Endoscopic } \\
\text { features }\end{array}$} & \multirow{2}{*}{$\begin{array}{l}\text { Esopha- } \\
\text { gus } \\
\text { Normal }\end{array}$} & \multirow{2}{*}{$\begin{array}{l}\text { GEJ } \\
\text { Normal }\end{array}$} & \multicolumn{3}{|l|}{ Fundus } & \multicolumn{3}{|l|}{ Body } & \multicolumn{3}{|c|}{ Antrum-pylorus } \\
\hline & & & All & Normal & $\begin{array}{l}\text { Erythe- } \\
\text { ma }\end{array}$ & All & Normal & $\begin{array}{l}\text { Erythe- } \\
\text { ma }\end{array}$ & All & Normal & $\begin{array}{l}\text { Erythe- } \\
\text { ma }\end{array}$ \\
\hline $\begin{array}{l}\text { Total biopsied, } \\
\mathrm{n}\end{array}$ & 73 & 19 & 33 & 24 & 9 & 188 & 117 & 72 & 239 & 97 & 142 \\
\hline $\begin{array}{l}\text { Definite man- } \\
\text { agement } \\
\text { change, } \\
\%(95 \% \mathrm{Cl})\end{array}$ & $\begin{array}{l}5 \\
(2-13)\end{array}$ & $\begin{array}{l}\mathbf{5} \\
(0.1-26)\end{array}$ & $\begin{array}{l}18 \\
(7-35)\end{array}$ & $\begin{array}{l}13 \\
(3-32)\end{array}$ & $\begin{array}{l}33 \\
(7-70)\end{array}$ & $\begin{array}{l}7 \\
(4-12)\end{array}$ & $\begin{array}{l}6 \\
(2-12)\end{array}$ & $\begin{array}{l}10 \\
(4-19)\end{array}$ & $\begin{array}{l}9 \\
(6-14)\end{array}$ & $\begin{array}{l}9 \\
(4-17)\end{array}$ & $\begin{array}{l}9 \\
(5-15)\end{array}$ \\
\hline $\begin{array}{l}\text { No manage- } \\
\text { ment change, } \\
\%(95 \% \mathrm{Cl})\end{array}$ & $\begin{array}{l}81 \\
(70-89)\end{array}$ & $\begin{array}{l}84 \\
(60-96)\end{array}$ & $\begin{array}{l}58 \\
(39-75)\end{array}$ & $\begin{array}{l}67 \\
(45-84)\end{array}$ & $\begin{array}{l}33 \\
(7-70)\end{array}$ & $\begin{array}{l}81 \\
(74-86)\end{array}$ & $\begin{array}{l}85 \\
(78-91)\end{array}$ & $\begin{array}{l}74 \\
(62-83)\end{array}$ & $\begin{array}{l}72 \\
(66-78)\end{array}$ & $\begin{array}{l}78 \\
(69-86)\end{array}$ & $\begin{array}{l}68 \\
(60-76)\end{array}$ \\
\hline \multicolumn{12}{|c|}{ Histopathologic findings } \\
\hline $\begin{array}{l}\text { Intestinal } \\
\text { metaplasia, } \\
\%(95 \% \mathrm{Cl})\end{array}$ & - & $\begin{array}{l}11 \\
(1-33)\end{array}$ & $\begin{array}{l}3 \\
(0.1-16)\end{array}$ & $\begin{array}{l}4 \\
(0.1-21)\end{array}$ & $\begin{array}{l}\mathbf{0} \\
(0-34)\end{array}$ & $\begin{array}{l}6 \\
(3-10)\end{array}$ & $\begin{array}{l}3 \\
(1-9)\end{array}$ & $\begin{array}{l}10 \\
(4-19)\end{array}$ & $\begin{array}{l}10 \\
(7-15)\end{array}$ & $\begin{array}{l}\mathbf{5} \\
(2-12)\end{array}$ & $\begin{array}{l}13 \\
(8-20)\end{array}$ \\
\hline $\begin{array}{l}\text { H. Pylori, } \\
\%(95 \% \mathrm{Cl})\end{array}$ & - & - & $\begin{array}{l}18 \\
(7-35)\end{array}$ & $\begin{array}{l}8 \\
(1-27)\end{array}$ & $\begin{array}{l}\mathbf{4 4} \\
(14-79)\end{array}$ & $\begin{array}{l}5 \\
(3-10)\end{array}$ & $\begin{array}{l}4 \\
(1-10)\end{array}$ & $\begin{array}{l}7 \\
(2-15)\end{array}$ & $\begin{array}{l}8 \\
(5-13)\end{array}$ & $\begin{array}{l}8 \\
(4-16)\end{array}$ & $\begin{array}{l}8 \\
(4-14)\end{array}$ \\
\hline
\end{tabular}

presence of duodenal ulcer. Twenty-three of $25 \mathrm{HP}$ results definitely changed management (the 2 that did not were for lack of evidence of follow-up). Of the 19 subjects in which IM was found in the stomach but HP was not, 3/19 (16\%) biopsies definitely changed management while $7 / 19$ (37\%) definitely did not. In the entire cohort, dysplasia or malignancy was found in only two non-targeted biopsies. These were in a patient with a history of MALToma in the stomach, and a patient with previous GEJ dysplasia status-post RFA, both undergoing surveillance for those conditions.

Eosinophilic infiltrate (not necessarily meeting criteria for EoE) was found in 9/73 non-targeted esophageal biopsies (12\%, Cl 6-22\%), all of which were performed for either surveillance of previous esophageal eosinophilia (7/9) and/or complaint of dysphagia (4/9). Eosinophilia accounted for 7/8 management changes (including "definite" and "uncertain significance") in the esophagus. All 3 subjects who experienced a management change based on a non-targeted biopsy of a normal GEJ had undergone endoscopy for an indication of Barrett's surveillance.

\section{Factors associated with management change}

Results of exploratory regression analyses of factors associated with management change from non-targeted gastric biopsies are shown in $>$ Table 5 . Older age, sex, non-white race, a preferred language that was not English, a history of positive HP test, fundus erythema and antral ulcer were associated with management change in bivariable analysis. Age greater than 50 and preferred language emerged as the most strongly associated variables in the multivariable models, reflecting the dependence of management changes on the detection of HP and GIM. Race was not included in any of the models with language, to avoid possible collinearity. Of note, the Sydney Protocol was not specifically mentioned in EGD reports, but 109 of 294 subjects could be considered to have received an approximation of this protocol, defined as at least 2 biopsies each from the antrum and body (specific curvature not reported). Receiving this surrogate Sydney Protocol was not a statistically significant predictor of management change, HP, or GIM in bivariable or multivariable analyses ( $P \geq 0.05$ for all comparisons). $>$ Supple- 
- Table 5 Patient and procedure characteristics associated with definite management change and histopathologic findings on gastric biopsy.

\begin{tabular}{|c|c|c|c|}
\hline Variable & Adjusted prevalence ratio ${ }^{1}$ & $P$ value & $95 \% \mathrm{Cl}$ \\
\hline \multicolumn{4}{|l|}{ Management change (28 events) } \\
\hline Age $>50$ & 6.03 & 0.003 & $1.87-19.45$ \\
\hline Non-English preferred language & 4.36 & $<0.001$ & $2.28-8.33$ \\
\hline \multicolumn{4}{|l|}{ H. Pylori ( 25 events) } \\
\hline Age $>50$ & 8.31 & 0.004 & $1.99-34.55$ \\
\hline Non-English preferred language & 4.12 & $<0.001$ & $2.03-8.38$ \\
\hline \multicolumn{4}{|l|}{ Intestinal metaplasia (28 events) } \\
\hline Age $>50$ & 3.51 & 0.012 & $1.38-8.93$ \\
\hline Antrum-pylorus erythema & 2.84 & 0.008 & $1.25-6.42$ \\
\hline
\end{tabular}

- Table 6 Estimated percentages of patients with clinically important outcome from non-targeted gastric biopsy, by strata.

\begin{tabular}{|c|c|c|}
\hline Combination of variables & Estimated percentage & $95 \% \mathrm{Cl}$ \\
\hline \multicolumn{3}{|l|}{ Management change } \\
\hline English-speaking, age $<50$ & $1.7 \%$ & $0.5-5.5 \%$ \\
\hline English-speaking, age $>50$ & $12.0 \%$ & $7.7-18.1 \%$ \\
\hline Non-English-speaking, age $<50$ & $12.0 \%$ & $3.3-35.6 \%$ \\
\hline Non-English-speaking, age $>50$ & $51.1 \%$ & $28.3-73.5 \%$ \\
\hline \multicolumn{3}{|l|}{ Helicobacter Pylori } \\
\hline English-speaking, age $<50$ & $1.2 \%$ & $0.3-4.8 \%$ \\
\hline English-speaking, age $>50$ & $11.1 \%$ & $7.0-17.1 \%$ \\
\hline Non-English-speaking, age $<50$ & $7.6 \%$ & $1.6-29.6 \%$ \\
\hline Non-English-speaking, age $>50$ & $46.2 \%$ & $24.0-70.0 \%$ \\
\hline \multicolumn{3}{|l|}{ Intestinal metaplasia } \\
\hline Age $<50$, no antral-pylorus erythema & $1.9 \%$ & $0.6-5.5 \%$ \\
\hline Age $>50$, no antral-pylorus erythema & $5.9 \%$ & $2.4-13.7 \%$ \\
\hline Age $<50$, antral-pylorus erythema & $7.3 \%$ & $3.4-14.7 \%$ \\
\hline Age $>50$, antral-pylorus erythema & $20.6 \%$ & $13.4-30.2 \%$ \\
\hline
\end{tabular}

mental Table 2 shows results of the sensitivity analysis of expanding the dependent variable to include management changes "of uncertain significance," although no additional associated variables were identified beyond those in $>$ Table 5 (at the level of $P<0.01$ ). To better assess the clinical significance of the factors identified in the multivariable analysis, - Table 6 displays stratified estimates of absolute proportions of patients that might experience each outcome.
Erythema was only significantly associated with certain outcomes when analyzed by region. Fundal erythema was associated with HP and therefore management change in the stomach in unadjusted analysis, but was not statistically significant in the multivariable model. Likewise, erythema of the body, fundus, antrum, or any gastric region ("pooled") were associated with GIM in any gastric region in unadjusted analysis, though only antral erythema was significant in the multivariable model at $P<0.01$. However, all of the associations with erythema are likely confounded by "number of non-targeted biopsies." That is, not only were a markedly higher proportion of erythematous 
stomachs biopsied as shown in $>$ Table 3, but these erythematous regions were also subject to higher total number of biopsy 'bites'. Adding the variable "number of non-targeted biopsies" to the models led to each "erythema" variable losing significance to below the cutoff (e.g. antral erythema to $P=0.091$ for any GIM). Additionally, there were no significant interactions between age or preferred language and erythema of the stomach ( $P>0.10$ for all comparisons), and the prevalence ratios of these covariates did not appreciably differ in samples restricted to those with only normal gastric mucosa versus those with erythema. Low number of management changes prohibited any significant regression models for the esophagus or GEJ.

\section{Discussion}

This study of 949 patients undergoing outpatient EGD at a high-volume endoscopy center demonstrates that there is wide variation in the practice of non-targeted biopsies of the stomach and esophagus, and such practice leads to changes in management in only a small proportion of patients. The primary reasons for changes in management based on biopsy results are HP in the stomach and eosinophilia in the esophagus. Exploratory analyses of this cohort revealed that age and preferred language were most associated with HP and consequently a definite change in management from a non-targeted gastric biopsy. Mild gastric erythema was associated with increased non-targeted biopsy, but a proportionate relationship to management was not evident, although this increased biopsy rate did yield a higher GIM detection rate.

Our results expand on what is known about the practice and yield of 'non-targeted' biopsies. We are not aware of any prior studies that examine the effect of mild or moderate gastroscopic "erythema" on likelihood of biopsy. Non-targeted biopsy of erythema occurred at a very high rate in the stomach. Given that our study did not involve independent visual verification of endoscopic descriptions, it is possible that the much less-often biopsied "normal" gastric mucosa in fact included small patches of "erythema" that the more conservative endoscopists classified as "normal" by virtue of their decision not to biopsy. In addition, the tertiary care referral setting may select a larger percentage of refractory cases, with increased need for diagnostic sensitivity leading to increased biopsy practice. Regardless, our study suggests that biopsy of mild "erythema" or "gastritis" in the gastric body or antrum does not lead to more management changes or diagnoses of HP than biopsy of "normal" mucosa, and that for most patients, such a mucosal appearance can be considered on the spectrum of normal. However, our exploratory analyses suggest that considering the patient's preferred language and age could increase the yield of such biopsies from $\sim 1 \%$ to over $30 \%$ ( $\triangleright$ Table 6 ), a clinically significant change.

The association of fundal erythema with the presence of HP and consequent management change ( $\triangleright$ Table 4 ) was an unexpected finding of the study. Biopsy of the fundus is not part of the Sydney protocol or any current guideline recommendations, though it may be reasonable when searching for HP in patients on PPIs, as acid suppression tends to drive HP higher up in the stomach. The overall rate of fundus biopsy was still expectedly low, and the wide confidence intervals for the effect of biopsy of fundal erythema raise the possibility that this association may still be the product of chance. The fact that biopsies of fundal erythema yielded a high rate of HP but never resulted in definite management change without a similarly impactful result from the body or antrum could indicate that fundal erythema is more of a specific than a sensitive macroscopic marker of a pan-gastritis. This hypothesis should be the target of further investigation.

Regarding specific histopathologic findings, our study is generally consistent with others. HP prevalence in Western populations ranges from $11.8 \%$ to $44.1 \%$ in endoscopies for dyspepsia [27-29], although these studies included biopsies of abnormal mucosa. Prevalence rates of gastric IM range from $3 \%$ to $22.9 \%$ [ $27-31$ ], with several studies also reporting associations of GIM with increasing age [27, 29-31]. In our study, GIM tended to appear more frequently in stomachs with antral erythema and consequent increased biopsy sampling, though these biopsy results were not more likely to result in definite management change. This likely reflects the lower consensus surrounding how exactly to deal with GIM found on non-targeted biopsy in a region of low gastric cancer incidence [12-14, 20].

Both HP and GIM vary substantially according to geography, ethnicity and economic factors [29-32]. In our dataset, nonEnglish preferred language was the best surrogate for being foreign-born, which may also correlate with socioeconomic status, less-developed water and sanitation systems during childhood, or other HP risk factors. That the overall rates of HP and GIM in our cohort are on the lower end of expected likely reflects the predominantly insured, Caucasian and African American, urban United States, tertiary care population, as well as the focus on biopsies of normal mucosa.

The prevalence rate of esophageal eosinophilia in esophageal biopsies without regard to mucosal appearance has been reported as $4.6 \%$ [33]. Our sample prevalence (12\%) approaches the markedly higher prevalence of esophageal eosinophilia from U.S. tertiary care centers (23\%) [34], perhaps due to inclusion of some patients who were undergoing surveillance biopsy for known or suspected EoE. In our study, all management changes occurred in subjects who either had a history of esophageal eosinophilia or presenting symptom of dysphagia, confirming that biopsy of the normal-appearing esophagus should rarely be considered outside of these situations [25].

The strengths of this study include that it provides realworld data regarding both non-targeted EGD biopsy practice patterns and outcomes in a developed country. The prevalence rates of the gastric and esophageal pathologies in question depend on geographic, ethnic and socioeconomic factors, and change over time. Endoscopic technologies also advance, allowing higher resolution for detection of gross pathology. Therefore, an updated, population-specific understanding of these pathologies is essential for the development of any modern quality improvement measures. This is the first study we are aware of to examine non-targeted biopsy across the entire spectrum of indications and patient characteristics. As such, it 
is positioned to inform the practice of biopsying non-specific lesions in an area of the upper gastroinestinal tract that is not related to the reason for endoscopy, and helps to confirm that this is a low-yield practice. Further, the outcome of "management change" is both patient-oriented and pragmatic, synthesizing the variability with which gastroenterologists interpret histologic findings (such as gastritis and GIM) into a measurable construct that reflects that finding's direct significance to the patient.

Some of these unique aspects of the study, however, are also limitations. Sample heterogeneity limits the power to analyze potentially relevant subgroups where non-targeted biopsy may be more appropriate (such as by indication, or index endoscopy, though neither of these were significantly associated with our primary outcome in regression analyses). We also did not have access to truly patient-oriented outcomes, such as symptom or quality-of-life ratings, or the rates of future ulcers or malignancies. The subjectivity of the primary outcome, while pragmatically reflecting current practice, is not guaranteed to reflect best practice, so that this report cannot serve as the sole basis for a judgment on the merits of non-targeted biopsy. Lastly, as data come from a single center with one pathology lab, the reporting of histopathologic findings and the effect of such findings on management may be biased toward local institutional culture.

We must also caution about over-generalization of our findings. We surveyed a random sample of procedures for a variety of indications, using white light endoscopy (WLE) in a low gastric cancer setting. Yield of biopsies may increase in gastric erythema noted to have additional features on narrow band (NBI) or other image-enhancement, $[35,36]$ or simply on a surveillance mapping endoscopy with careful mucosal inspection under high resolution, magnified WLE. Many of our study's EGD reports did not distinguish between diffuse and localized erythema either, the latter of which may be more likely to harbor dysplasia or even early gastric cancer [36] and therefore warrant biopsy or resection, especially in regions of higher gastric cancer incidence.

Despite these limitations, our study's findings support the following conclusions. Non-targeted biopsies of the esophagus should usually only be undertaken for patients with dysphagia or known esophageal eosinophilia, because they are otherwise unlikely to affect management. Mild gastric erythema on WLE should not be regarded as qualitatively different from normal mucosa with regard to HP, although its relationship to the presence of GIM remains uncertain. It may be extrapolated that if one is searching for HP, it is advisable to still adhere to the Sydney protocol for standard WLE, or else consider using image enhancement such as NBI. Identification of patient characteristics predictive of occult HP is difficult in low prevalence populations such as this U.S. academic center, but surrogates (for our center) of being born in a less developed country (non-Englishspeaking language) and age $>50$ showed the strongest, most clinically significant associations, consistent with the observations of guidelines committees [15, 16, 18-20].

We practice in an increasingly cost- and quality-conscious healthcare environment. Strategies that serve only to increase the sensitivity of a diagnostic method have not always proven cost-effective or even efficacious for improving patient-oriented outcomes. We must rigorously evaluate such methods, and the upper $\mathrm{Gl}$ tract is no exception. At present, biopsy of non-specific findings during EGD should be based primarily on the patient characteristics and procedure indication, rather than the appearance of erythema under unenhanced, white light. Prospective, multi-center studies are warranted to compare non-targeted biopsy to other strategies such as image-enhanced endoscopy for appropriate indications. These may identify additional patient and mucosal characteristics predictive of significant histopathologic findings, as well as examine cost-effectiveness and symptomatic outcomes. Such studies could greatly inform quality improvement in upper endoscopy.

\section{Acknowledgements}

Dr. Dougherty was supported in part by a grant from the $\mathrm{NIH}$ (T32 DK007634).

\section{Competing interests}

None

\section{References}

[1] Cosgrove JC, Walke JD, Anderson TD et al. Action needed to address higher use of anatomic pathology services by providers who self-refer. GAO; 2013: http://www.gao.gov/products/GAO-13-445 [Accessed July 16, 2017]

[2] Allen JI, Katzka D, Robert M et al. American Gastroenterological Association Institute Technical Review on the Role of Upper Gastrointestinal Biopsy to Evaluate Dyspepsia in the Adult Patient in the Absence of Visible Mucosal Lesions. Gastroenterology 2015; 149: 1088 1118

[3] Carabotti M, Lahner E, Porowska B et al. Are clinical features able to predict Helicobacter pylori gastritis patterns? Evidence from tertiary centers Intern Emerg Med 2014; 9: 841 - 845

[4] Johnsen R, Bernersen B, Straume B et al. Prevalences of endoscopic and histological findings in subjects with and without dyspepsia. BM] 1991; 302: 749-752

[5] Moayyedi P. Helicobacter pylori eradication for functional dyspepsia: what are we treating? Comment on "Helicobacter pylori eradication in functional dyspepsia”. Arch Intern Med 2011; 171: 1936- 1937

[6] Makris N, Crott R, Fallone CA et al. Cost-effectiveness of routine endoscopic biopsies for Helicobacter pylori detection in patients with non-ulcer dyspepsia. Gastrointest Endosc 2003; 58: 14-22

[7] de Vries AC, Haringsma J, de Vries RA et al. Biopsy strategies for endoscopic surveillance of pre-malignant gastric lesions. Helicobacter 2010; 15: 259-264

[8] El-Zimaity HM, Graham DY. Evaluation of gastric mucosal biopsy site and number for identification of Helicobacter pylori or intestinal metaplasia: role of the Sydney System. Hum Pathol 1999; 30: $72-77$

[9] Breslin NP, Thomson AB, Bailey RJ et al. Gastric cancer and other endoscopic diagnoses in patients with benign dyspepsia. Gut 2000; 46: $93-97$

[10] Cadman B, Dixon MF, Wyatt JI. Value of routine, non-targeted biopsies in the diagnosis of gastric neoplasia. J Clin Pathol 1997; 50: 832 - 834 
[11] Fiorenza JP, Tinianow AM, Chan WW. The Initial Management and Endoscopic Outcomes of Dyspepsia in a Low-Risk Patient Population. Digestive Diseases and Sciences 2016; 61: 2942-2948

[12] Areia M, Carvalho R, Cadime AT et al. Screening for gastric cancer and surveillance of premalignant lesions: a systematic review of cost-effectiveness studies. Helicobacter 2013; 18: 325 - 337

[13] O'Connor A, McNamara D, O'Morain CA. Surveillance of gastric intestinal metaplasia for the prevention of gastric cancer. Cochrane Database Syst Rev 2013; 9: CD009322

[14] Zullo A, Hassan C, Repici A et al. Intestinal metaplasia surveillance: searching for the road-map. World J Gastroenterol 2013; 19: 1523 1526

[15] Evans JA, Chandrasekhara V, Chathadi KV et al. The role of endoscopy in the management of premalignant and malignant conditions of the stomach. Gastrointest endosc 2015; 82: 1 -8

[16] Chey WD, Wong BC. Practice Parameters Committee of the American College of Gastroenterology. American College of Gastroenterology guideline on the management of Helicobacter pylori infection. Am J Gastroenterol 2007; 102: 1808-1825

[17] Talley NJ, Vakil N. Practice Parameters Committee of the American College of Gastroenterology. Guidelines for the management of dyspepsia. Am J Gastroenterol 2005; 100: 2324-2337

[18] Ikenberry SO, Harrison ME, Lichtenstein D et al. The role of endoscopy in dyspepsia. Gastrointest Endosc 2007; 66: 1071-1075

[19] Yang Y-X, Brill J, Krishnan P et al. American Gastroenterological Association Institute Guideline on the Role of Upper Gastrointestinal Biopsy to Evaluate Dyspepsia in the Adult Patient in the Absence of Visible Mucosal Lesions. Gastroenterology149: 1082-1087

[20] Dinis-Ribeiro M, Areia M, de Vries AC et al. Management of precancerous conditions and lesions in the stomach (MAPS): guideline from the European Society of Gastrointestinal Endoscopy (ESGE), European Helicobacter Study Group (EHSG), European Society of Pathology (ESP), and the Sociedade Portuguesa de Endoscopia Digestiva (SPED). Endoscopy 2012; 44: 74-94

[21] Katz PO, Gerson LB, Vela MF. Guidelines for the diagnosis and management of gastroesophageal reflux disease. Am J Gastroenterol 2013; 108: 308 -328; quiz 329

[22] Kahrilas PJ, Shaheen N], Vaezi MF et al. American Gastroenterological Association Medical Position Statement on the management of gastroesophageal reflux disease. Gastroenterology 2008; 135: $1383-$ 1391, 1391 e1381-1385

[23] Dellon ES, Gonsalves N, Hirano I et al. ACG clinical guideline: Evidenced based approach to the diagnosis and management of esophageal eosinophilia and eosinophilic esophagitis (EoE). Am J Gastroenterol 2013; 108: 679-692; quiz 693
[24] Kim HP, Vance RB, Shaheen NJ et al. The prevalence and diagnostic utility of endoscopic features of eosinophilic esophagitis: a meta-analysis. Clin Gastroenterol Hepatol 2012; 10: 988 - 996 e985

[25] Foroutan M, Norouzi A, Molaei $\mathrm{M}$ et al. Eosinophilic esophagitis in patients with refractory gastroesophageal reflux disease. Dig Dis Sci 2010; 55: $28-31$

[26] Dubin SM, Kwong WT, Kalmaz D et al. Low yield of routine duodenal biopsies for evaluation of abdominal pain. World J Gastroenterol 2015; 21: $7495-7499$

[27] Eriksson NK, Karkkainen PA, Farkkila MA et al. Prevalence and distribution of gastric intestinal metaplasia and its subtypes. Dig Liver Dis 2008; 40: $355-360$

[28] Lash JG, Genta RM. Adherence to the Sydney System guidelines increases the detection of Helicobacter gastritis and intestinal metaplasia in 400738 sets of gastric biopsies. Aliment Pharmacol Ther 2013; 38: 424-431

[29] Lahner E, Zullo A, Hassan C et al. Detection of gastric precancerous conditions in daily clinical practice: a nationwide survey. Helicobacter 2014; 19: 417-424

[30] Simko V, Anand N, Ginter E. Gastric intestinal metaplasia - age, ethnicity and surveillance for gastric cancer. Bratisl Lek Listy 2015; 116: $3-8$

[31] den Hoed CM, van Eijck BC, Capelle LG et al. The prevalence of premalignant gastric lesions in asymptomatic patients: Predicting the future incidence of gastric cancer. European Journal of Cancer 2011; 47: $1211-1218$

[32] Marques-Silva L, Areia M, Elvas L et al. Prevalence of gastric precancerous conditions: a systematic review and meta-analysis. European Journal of Gastroenterology \& Hepatology 2014; 26: 378-387

[33] Jensen ET, Eluri S, Lebwohl B et al. Increased risk of esophageal eosinophilia and eosinophilic esophagitis in patients with active celiac disease on biopsy. Clin Gastroenterol Hepatol 2015; 13 : 081426 1431

[34] Dellon ES, Speck O, Woodward K et al. Distribution and variability of esophageal eosinophilia in patients undergoing upper endoscopy. Mod Pathol 2015; 28: 383-390

[35] Muto M, Yao K, Kaise M et al. Magnifying endoscopy simple diagnostic algorithm for early gastric cancer (MESDA-G). Digestive Endoscopy 2016; 28: 379-393

[36] Yao K. The endoscopic diagnosis of early gastric cancer. Annals of Gastroenterology: Quarterly Publication of the Hellenic Society of Gastroenterology 2013; 26: 11-22 
Supplemental Table 1 Indications associated with non-targeted biopsy by region.

\begin{tabular}{|c|c|c|c|}
\hline & Adjusted prevalence ratio ${ }^{1}$ & P value & $95 \% \mathrm{Cl}$ \\
\hline \multicolumn{4}{|l|}{ Stomach (all regions) } \\
\hline Dyspepsia/abdominal pain & 2.10 & $<0.001$ & $1.74-2.53$ \\
\hline Nausea & 1.43 & 0.006 & $1.11-1.85$ \\
\hline Barrett's surveillance & 0.50 & 0.022 & $0.28-0.90$ \\
\hline Planned procedure & 0.40 & 0.033 & $0.17-0.93$ \\
\hline \multicolumn{4}{|l|}{ Esophagus } \\
\hline Dysphagia/odynophagia & 5.55 & $<0.001$ & $3.63-8.48$ \\
\hline GERD & 1.86 & 0.010 & $1.16-2.98$ \\
\hline Chest pain & 4.09 & $<0.001$ & $2.03-8.21$ \\
\hline Surveillance of esophageal eosinophilia & 2.52 & 0.003 & $1.37-4.64$ \\
\hline \multicolumn{4}{|l|}{ GEJ } \\
\hline Barrett's surveillance & 4.23 & $<0.001$ & $2.72-6.58$ \\
\hline GERD & 2.31 & $<0.001$ & $1.57-3.39$ \\
\hline Chest Pain & 2.31 & 0.012 & $1.20-4.47$ \\
\hline Dyspepsia & 1.80 & 0.013 & $1.13-2.86$ \\
\hline \multicolumn{4}{|c|}{$\begin{array}{l}\mathrm{CI} \text {, confidence interval; GEJ, gastroesophageal junction; GERD, gastroesophageal reflux disease } \\
1 \text { Using a modified Poisson regression with robust variance, we adjusted for age, sex, and a history of positive } H \text {. Pylori test, inflammatory bowel disease, foregut } \\
\text { surgery, or previousEsophagogastroduodenoscopy }\end{array}$} \\
\hline
\end{tabular}

- Supplemental Table 2 Patient and procedure characteristics associated with "any" management change ${ }^{1}$ from gastric biopsy (events $\left.=54\right)^{2}$.

\begin{tabular}{|l|l|l|l|}
\hline Variable & Adjusted Prevalence Ratio & P value & $\mathbf{9 5 \% ~ C l}$ \\
\hline Non-English preferred language & 2.76 & $<0.001$ & $1.65-4.61$ \\
\hline Indication dysphagia/ odynophagia & 0.39 & 0.089 & $0.13-1.16$ \\
\hline Indication change in bowel habits & 0.30 & 0.094 & $0.08-1.23$ \\
\hline Duodenitis & 2.83 & 0.014 & $1.24-6.48$ \\
\hline Fundus erythema & 2.14 & 0.013 & $1.18-3.92$ \\
\hline
\end{tabular}

$\mathrm{Cl}$, Confidence interval

1 Includes "definite" management change, and change of "uncertain significance."

${ }^{2}$ As noted in bold, only one variable (language) survived the pre-specified cutoff of $P<0.01$. However, given that this is a sensitivity analysis of an exploratory analysis, with sufficiently higher number of outcomes to permit additional variables in the model, the results of a model with a more liberal threshold are displayed here for the interested reader. Models were also created substituting erythema of body, antrum, or "any" gastric region as the "erythema" variable, but fundus erythema was the only site with a $P$ value $<0.20$. Binomial regression model did not converge, so we used a modified Poisson regression, with robust variance, for this analysis. 